\title{
DUS Characterization of Germplasm Accessions in Foxtail Millet (Setaria italica (L.) Beauv.)
}

\author{
K. Amarnath ${ }^{1 *}$, A.V.S. Durga Prasad ${ }^{1}$ and C.V. Chandra Mohan Reddy ${ }^{2}$ \\ ${ }^{1}$ Department of Genetics and Plant Breeding, Agricultural College, \\ Mahanandi -518 502, A.P., India \\ ${ }^{2}$ RARS, Nandyal - 518 501, A.P., India \\ *Corresponding author
}

\section{A B S T R A C T}

\section{Keywords}

DUS Descriptors,

Foxtail millet,

Qualitative traits,

Quantitative traits,

Morphological

characterization

Article Info

Accepted:

18 January 2019

Available Online:

10 February 2019
In the present study, 50 germplasm accessions of foxtail millet [Setaria italica (L.) Beauv.] were evaluated based on DUS descriptors for six morphological (qualitative) traits viz., plant growth habit, plant pigmentation at auricle, inflorescence shape, inflorescence compactness, lobe compactness, seed colour and six quantitative characters viz., flag leaf: blade length $(\mathrm{cm})$, flag leaf: blade width $(\mathrm{cm})$, peduncle length $(\mathrm{cm})$, peduncle exertion, panicle length $(\mathrm{cm})$ and plant height at maturity $(\mathrm{cm})$. All the germplasm accessions were characterized into different groups based on each character. The results revealed significant variation among the germplasm accessions for all the characters studied implying that morphological characterization of the foxtail millet germplasm accessions had enormous genetic diversity that will assign them key diagnostic features facilitating selection process, enabling the plant breeders in proper documentation to protect them from any possible infringement.

\section{Introduction}

Foxtail millet (Setaria italica (L.) Beauv.), an annual autogamous diploid $(2 \mathrm{n}=18)$ small millet is a photosynthetically efficient $\mathrm{C}_{4}$ monocot belonging to the family, Poaceae. Out of $80 \mathrm{k}$ ha. grown in India, this crop is cultivated in an area of $51 \mathrm{k}$ ha. in Andhra Pradesh with a productivity of $945 \mathrm{~kg}$ ha-1 compared to national terms of $900 \mathrm{~kg}$ ha-1 (Annual report, 2016-17). This millet grains offers an excellent source of quality proteins (leucine and methionine), $\beta$-carotene, minerals ( $\mathrm{Ca}, \mathrm{Fe}, \mathrm{K}, \mathrm{Mg}$ and $\mathrm{Zn}$ ), antioxidants, dietary fibre, phytochemicals, vitamins (thiamine, riboflavin and niacin) and have low glycemic index, a requisite for healthy human diet (Murugan and Nirmalakumari, 2006). Besides, its small genome size of $\sim 515 \mathrm{Mb}$ ( $\mathrm{Li}$ and Brutnell, 2011) provides ample scope for molecular and genetic research as a 'model crop' to understand deeper insights into plant biology. The 'Protection of Plant Varieties and 
Farmers' Right Act, 2001 (PPV \& FR Act, 2001) of India encourages public/private investment in research and development of new plant varieties by giving protection to the new plant genotypes against unauthorized multiplication of seeds or propagating materials for a specific period. In addition, the act insists on DUS characterization of extant, farmers and new varieties and recommends the registration of varieties for atleast any one specific novel character.

The new foxtail millet genotypes will be protected under the PPV \& FR Act after confirming DUS testing through the comparison of new and existing varieties in similar environment for a set of morphological characters. Characterization of these diverse germplasm accessions for their morphological characteristics holds immense potential for their objective utilization in the breeding programme. Further they serve as an indispensable tool in selecting varieties or lines based on agronomical, morphological, genetic or physiological characters. Therefore, the present investigation was conducted to characterize the foxtail millet germplasm accessions for selected DUS characters, yield and yield attributing traits.

\section{Materials and Methods}

\section{Plant materials and field trails}

The experimental material for the present study comprised of 50 foxtail millet germplasm accessions chosen from 1038 accessions maintained at Regional Agricultural Research Station (RARS), Nandyal, Andhra Pradesh, India. These germplasm accessions were raised in a field trail of randomized complete block design (RCBD), replicated thrice with a plot size of $13 \mathrm{~m} \times 15 \mathrm{~m}$ per each germplasm accession, with a spacing of $22.5 \mathrm{~cm}$ and $10 \mathrm{~cm}$ between rows and plants, respectively during kharif, season at RARS, Nandyal. Standard cultural and agronomic practices were adopted to raise a healthy crop. The experimental field was protected from bird damage during grain filling stage by installing bird scarers. Based on attainment of harvestable maturity, the foxtail millet germplasm accessions studied were harvested at varied intervals (Table 1).

\section{Data recording and analysis}

The observations based on DUS descriptors for six morphological (qualitative) traits (Table 2) viz., plant growth habit, plant pigmentation at auricle, inflorescence shape, inflorescence compactness, lobe compactness, seed colour and six quantitative characters (Table 3) viz., flag leaf: blade length $(\mathrm{cm})$, flag leaf: blade width $(\mathrm{cm})$, peduncle length $(\mathrm{cm})$, peduncle exertion, panicle length $(\mathrm{cm})$ and plant height at maturity $(\mathrm{cm})$ were recorded for each germplasm accession on five randomly selected plants per replication.

The range, mean and coefficient of variations for six quantitative characters were computed.

\section{Results and Discussion}

\section{Qualitative descriptor}

Characterization of the qualitative traits revealed a wide variation among the accession (Table 4). Each descriptor is categorized based on morphological descriptor and is presented as key for identification (Flow charts) (Fig. 3).

\section{Plant growth habit}

In the present study two kinds of Plant growth habit was noticed: Among 50 Italian millet germplasm accessions, Erect was dominant (41) over Decumbent (9). Similar results were reported by Reddy et al., (2006), Reddy et al., (2009) for erect type of growth habit (Fig. 1). 


\section{Plant pigmentation at auricle}

Two types of plant pigmentation at auricle was occurred in Italian millet germplasm accessions and categorized as Pigmented types and Non-Pigmented.

The Non-pigmented types (42) were dominant over Pigmented types (8). Similar result was obtained by Banu et al., (2015) for this trait

\section{Inflorescence shape}

In the present study, inflorescence shapes of genotypes were categorized into three groups viz., oblong (27), cylindrical (14) and pyramidal (9). Similar classification was reported by Radha et al., (2014) and Ahmed et al., (2017) for this trait.

\section{Inflorescence compactness}

Based on this trait, the germplasm accessions were grouped into three types i.e., loose (3), medium (44) and compact (3).

The results found were in conformity with the findings of Reddy et al., (2006), Radha et al., (2014).

\section{Lobe compactness}

Based on the lobe compactness, germplasm accessions were grouped into three classes loose (3), medium (44) and compact (3). Similar results were obtained by Radha $e t$ al., (2014), Ahmed et al., (2017).

\section{Seed colour}

Based on seed colour, the germplasm accessions were categorized as light yellow (34), yellow (13), white (1), orange (1) and red (1). Contrarily, Reddy et al., (2006), Radha et al., (2014) and Banu et al., (2017) reported that yellow seeds were more dominant.

\section{Quantitative descriptor}

Range, mean and coefficient of variations for six quantitative characters were shown in Table 5 and Figure 2.

\section{Flag leaf: Blade length (cm)}

Majority (44) of the germplasm accessions exhibited medium while the remaining were long for the trait, flag leaf blade length.

This result is in accordance with the findings of Ahmed et al., (2017) and Nehra et al., (2017).

\section{Flag leaf: Blade width (cm)}

Medium blade width for flag leaf was observed in all the germplasm accessions. Similar finding was also reported by Ahmed et al., (2017).

\section{Peduncle length $(\mathrm{cm})$}

All the germplasm accessions were characterized either medium (28) or long (22) for this trait.

This result is in consonance with the findings of Reddy et al., (2006) and Amgai et al., (2011).

\section{Peduncle exertion}

Among the germplasm accessions studied, the trait peduncle exertion was observed to be either long (47) or short (03). Similar result was reported by Amgai et al., (2011) for this character.

\section{Plant height at maturity $(\mathrm{cm})$}

Most of the germplasm accessions were tall (44) accessions while few were medium (6). Similar result was obtained by Nehra et al., (2016). 
Int.J.Curr.Microbiol.App.Sci (2019) 8(2): 2410-2422

Table.1 Details of 50 foxtail millet germplasm accessions utilized for study

\begin{tabular}{|c|c|c|}
\hline S. No & Germplasm accession & Parentage \\
\hline 1 & SiA 3085 & Selection from SiA 2644 from farmers field \\
\hline 2 & SiA 3156 & Pureline selection from SiA 2871 \\
\hline 3 & SiA 3221 & SiA 3075 x ISC 379 \\
\hline 4 & SiA 3322 & Selection from ISC 1161 \\
\hline 5 & SiA 3327 & Selection from ISC 1076 \\
\hline 6 & SiA 3328 & Selection from ISC 748 \\
\hline 7 & SiA 3333 & Selection from ISC 909 \\
\hline 8 & SiA 3335 & Selection from ISC 745 \\
\hline 9 & SiA 3340 & Selection from ISC 362 \\
\hline 10 & SiA 3346 & Selection from ISC 160 \\
\hline 11 & SiA 3347 & Selection from ISC 1162 \\
\hline 12 & SiA 3354 & Selection from ISC 18 \\
\hline 13 & SiA 3355 & Selection from ISC 869 \\
\hline 14 & SiA 3363 & Selection from ISC 1134 \\
\hline 15 & SiA 3364 & Selection from ISC 267 \\
\hline 16 & SiA 3367 & Selection from ISC 1118 \\
\hline 17 & SiA 3369 & Selection from ISC 1177 \\
\hline 18 & SiA 3375 & Selection from ISC 838 \\
\hline 19 & SiA 3376 & Selection from ISC 796 \\
\hline 20 & SiA 3377 & Selection from ISC 254 \\
\hline 21 & SiA 3378 & Selection from ISC 525 \\
\hline 22 & SiA 3381 & Selection from ISC 398 \\
\hline 23 & SiA 3382 & Selection from ISC 403 \\
\hline 24 & SiA 3383 & Selection from ISC 200 \\
\hline 25 & SiA 3384 & Selection from ISC 480 \\
\hline 26 & SiA 3386 & Selection from ISC 907 \\
\hline 27 & SiA 3389 & Selection from ISC 507 \\
\hline 28 & SiA 3390 & Selection from ISC 828 \\
\hline 29 & SiA 3392 & Selection from ISC 717 \\
\hline 30 & SiA 3393 & Selection from ISC 375 \\
\hline 31 & $\mathrm{SiA} 3394$ & Selection from ISC 792 \\
\hline 32 & SiA 3395 & Selection from ISC 931 \\
\hline 33 & SiA 3396 & Selection from ISC 846 \\
\hline 34 & SiA 3397 & Selection from ISC 917 \\
\hline 35 & SiA 3398 & Selection from ISC 179 \\
\hline 36 & SiA 3399 & Selection from ISC 237 \\
\hline 37 & SiA 3400 & Selection from ISC 388 \\
\hline 38 & $\mathrm{SiA} 3401$ & Selection from ISC 735 \\
\hline 39 & $\mathrm{SiA} 3403$ & Selection from ISC 842 \\
\hline 40 & $\mathrm{SiA} 3404$ & Selection from ISC 195 \\
\hline 41 & SiA 3405 & Selection from ISC 364 \\
\hline 42 & SiA 3407 & Selection from ISC 96 \\
\hline 43 & $\mathrm{SiA} 3447$ & Selection from ISC 1704 \\
\hline 44 & SiA 3381 & Selection from ISC 936 \\
\hline 45 & Srilakshmi & Pureline selection from local \\
\hline 46 & Suryanandi & Pureline selection from SiA 1244 \\
\hline 47 & Prasad & Selection from Dronachalam village \\
\hline 48 & Narasimharaya & $\mathrm{SiA} 805 \times \mathrm{SiA} 242$ \\
\hline 49 & Krishnadevaraya & $\mathrm{SiA} 326 \times \mathrm{SiA} 242$ \\
\hline 50 & ISC 347 & ICRISAT gene bank \\
\hline
\end{tabular}


Table.2 Characteristics of six morphological traits

\begin{tabular}{|c|c|c|c|}
\hline S.No & Character & Status & Stage of Observation \\
\hline \multirow[t]{2}{*}{1} & \multirow[t]{2}{*}{ Plant growth habit } & Erect & \multirow[t]{2}{*}{ Flowering } \\
\hline & & Decumbent & \\
\hline \multirow[t]{2}{*}{2} & \multirow[t]{2}{*}{ Plant: Pigmentation at auricle } & Absent & \multirow[t]{2}{*}{ Flowering } \\
\hline & & Present & \\
\hline \multirow[t]{3}{*}{3} & \multirow[t]{3}{*}{ Inflorescence: Shape } & Oblong & \multirow[t]{3}{*}{ Flowering } \\
\hline & & Pyramidal & \\
\hline & & Cylindrical & \\
\hline \multirow[t]{3}{*}{4} & \multirow[t]{3}{*}{ Inflorescence Compactness } & Loose & \multirow{3}{*}{ Dough } \\
\hline & & Medium & \\
\hline & & Compact & \\
\hline \multirow[t]{3}{*}{5} & \multirow[t]{3}{*}{ Lobe compactness } & Loose & \multirow[t]{3}{*}{ Maturity } \\
\hline & & Medium & \\
\hline & & Compact & \\
\hline \multirow[t]{5}{*}{6} & \multirow[t]{5}{*}{ Seed: Colour } & Orange & \multirow{5}{*}{ Maturity } \\
\hline & & Red & \\
\hline & & Yellow & \\
\hline & & Light Yellow & \\
\hline & & Straw & \\
\hline
\end{tabular}

Table.3 Characteristics of six quantitative characters

\begin{tabular}{|c|c|c|c|c|}
\hline S.No & Character & Status & Note & Stage of Observation \\
\hline \multirow[t]{3}{*}{1} & \multirow[t]{3}{*}{ Flag leaf: Blade length $(\mathrm{cm})$} & Short $(<20)$ & 1 & \multirow{3}{*}{ Flowering } \\
\hline & & Medium (20-35) & 9 & \\
\hline & & Long $(<35)$ & & \\
\hline \multirow[t]{3}{*}{2} & \multirow{3}{*}{ Flag leaf: Blade width $(\mathrm{cm})$} & Narrow $(<1.5)$ & 1 & \multirow{3}{*}{ Flowering } \\
\hline & & Medium (1.5-3.0) & & \\
\hline & & Long $(>3.0)$ & 9 & \\
\hline \multirow[t]{4}{*}{3} & \multirow[t]{4}{*}{ Peduncle length (cm) } & Short $(<20)$ & 1 & \multirow{4}{*}{ Flowering } \\
\hline & & Medium(20.0-30.0) & & \\
\hline & & Long $(30.0-40)$ & 3 & \\
\hline & & Very long $(>40)$ & 5 & \\
\hline \multirow[t]{4}{*}{4} & \multirow[t]{4}{*}{ Peduncle exertion } & Short $(<20)$ & 3 & \multirow[t]{4}{*}{ Dough } \\
\hline & & Medium(20.0-30.0) & & \\
\hline & & Long $(30.0-40)$ & 5 & \\
\hline & & Very long $(>40)$ & 7 & \\
\hline \multirow[t]{3}{*}{4} & \multirow[t]{3}{*}{ Plant height at maturity } & Short $(<80)$ & 3 & \multirow[t]{3}{*}{ Maturity } \\
\hline & & Medium (80-120) & 5 & \\
\hline & & Compact $(>120)$ & 7 & \\
\hline \multirow[t]{3}{*}{6} & \multirow[t]{3}{*}{ Panicle length } & Short $(<10)$ & \multirow[b]{3}{*}{-} & \multirow{3}{*}{ Maturity } \\
\hline & & Medium(10.0-15.0) & & \\
\hline & & Long $(>15)$ & & \\
\hline
\end{tabular}


Table.4 Morphological characterization in 50 foxtail millet germplasm accessions

\begin{tabular}{|c|c|c|c|c|}
\hline S.No. & Name of the descriptor & Descriptor state & No. of accesssions & $\begin{array}{c}\text { Frequency } \\
(\%)\end{array}$ \\
\hline \multirow[t]{2}{*}{1} & \multirow[t]{2}{*}{ Plant growth habit } & Erect & 41 & 82 \\
\hline & & Decumbent & 9 & 18 \\
\hline \multirow[t]{2}{*}{2} & \multirow[t]{2}{*}{ Plant pigmentation at auricle } & Pigmented & 8 & 16 \\
\hline & & Non Pigmeneted & 42 & 84 \\
\hline \multirow[t]{3}{*}{3} & \multirow[t]{3}{*}{ Inflorescence shape } & Oblong & 27 & 54 \\
\hline & & Pyramidal & 9 & 18 \\
\hline & & Cylindrical & 14 & 28 \\
\hline \multirow[t]{3}{*}{4} & \multirow[t]{3}{*}{ Inflorescence compactness } & Loose & 3 & 6 \\
\hline & & Medium & 44 & 88 \\
\hline & & Compact & 3 & 6 \\
\hline \multirow[t]{3}{*}{5} & \multirow[t]{3}{*}{ Lobe compactness } & Loose & 3 & 18 \\
\hline & & Medium & 44 & 72 \\
\hline & & Compact & 3 & 10 \\
\hline \multirow[t]{6}{*}{6} & \multirow[t]{6}{*}{ Seed colour } & Yellow & 13 & 26 \\
\hline & & White & 1 & 2 \\
\hline & & Orange & 1 & 2 \\
\hline & & Red & 1 & 2 \\
\hline & & Light Yellow & 34 & 68 \\
\hline & & Yellow & 13 & 26 \\
\hline
\end{tabular}

Table.5 Quantitative variations for six descriptors of foxtail millet germplasm accessions

\begin{tabular}{|l|c|c|c|}
\hline \multicolumn{1}{|c|}{ Descriptors } & Mean & Range & CV (\%) \\
\hline Flag leaf: Blade length (cm) & 32.19 & $25.60-37.80$ & 9.97 \\
\hline Flag leaf: Blade width (cm) & 1.86 & $1.29-2.14$ & 7.42 \\
\hline Peduncle length (cm) & 29.80 & $23.10-37.73$ & 7.38 \\
\hline Peduncle exertion & 15.19 & $9.77-23.00$ & 16.32 \\
\hline Plant height at maturity (cm) & 131.87 & $101.73-153.33$ & 6.31 \\
\hline Panicle length (cm) & 18.07 & $10.55-22.10$ & 12.34 \\
\hline
\end{tabular}


Fig.1 Qualitative characters

1. Plant growth habit

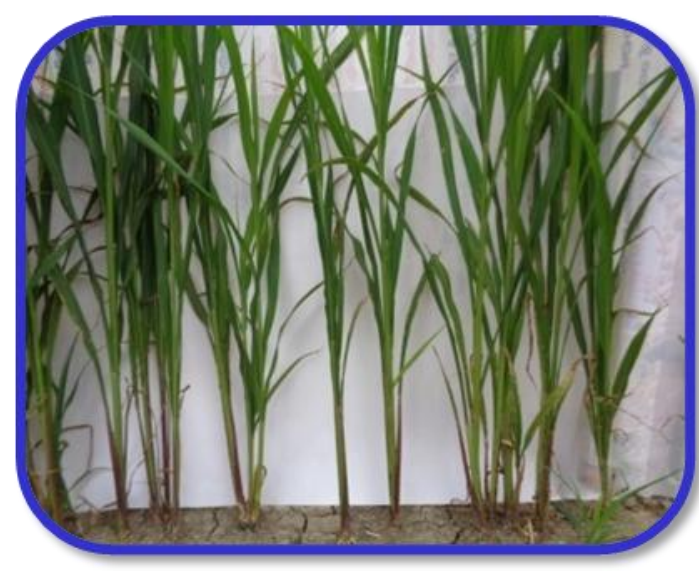

Erect

2. Plant pigmentation at auricle

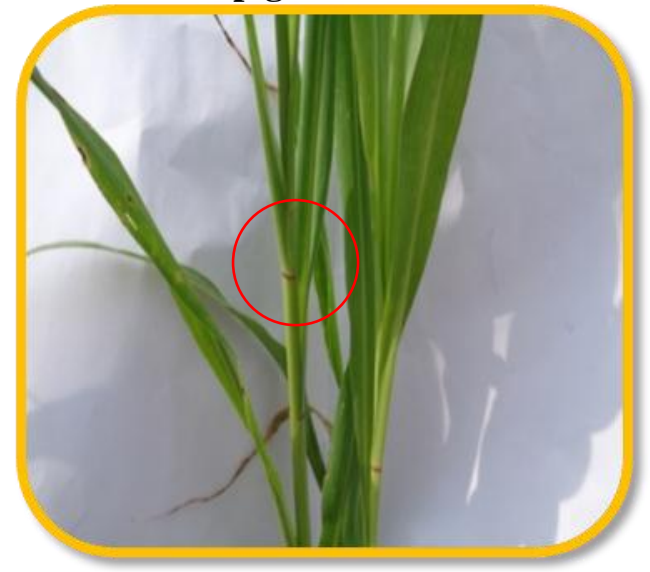

Pigmented

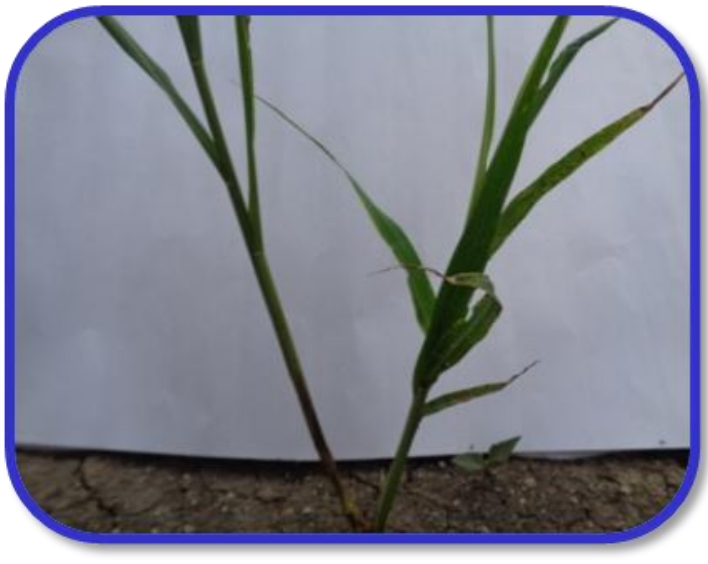

Decumbent

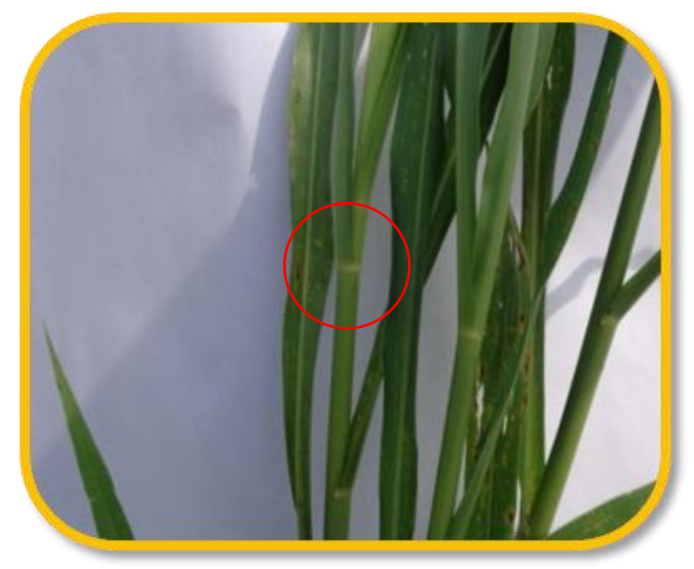

Non- pigmented

3. Inflorescence shape
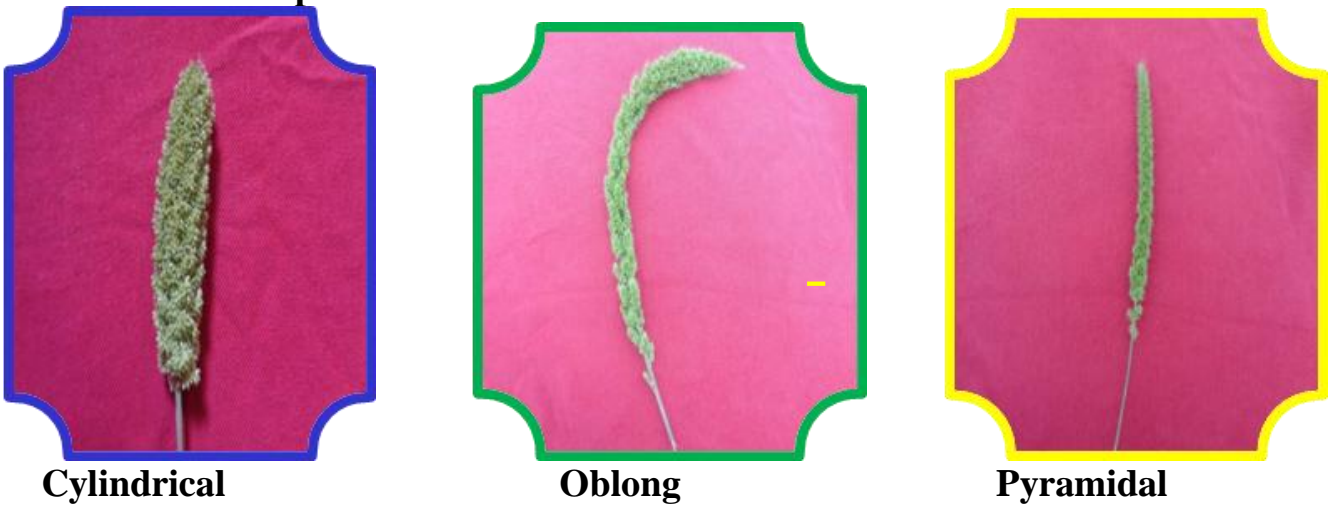
4. Inflorescence compactness

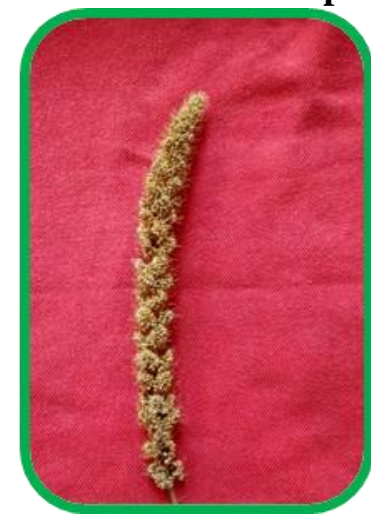

Loose

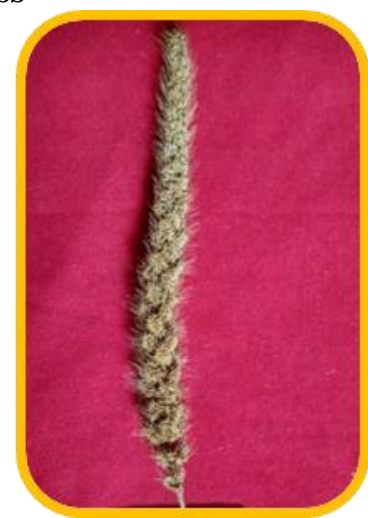

Medium

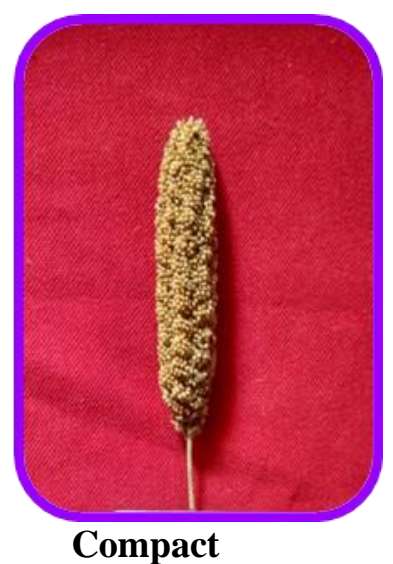

5. Lobe compactness

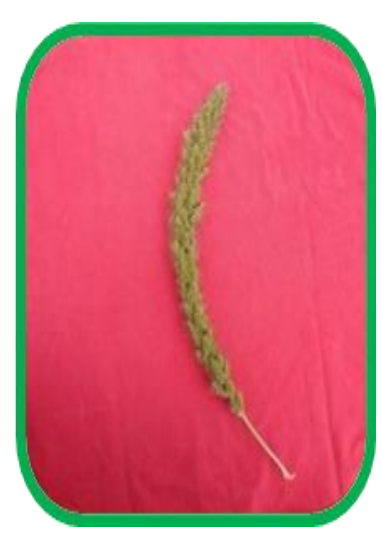

Loose

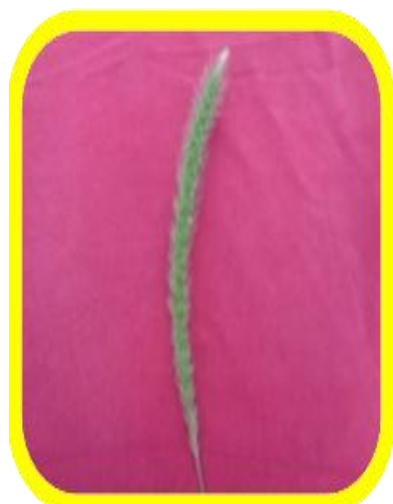

Medium

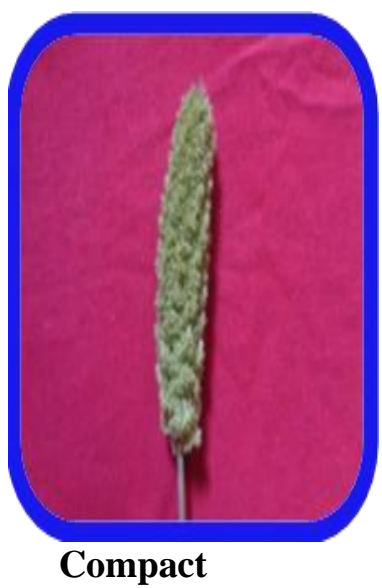

6. Seed colour

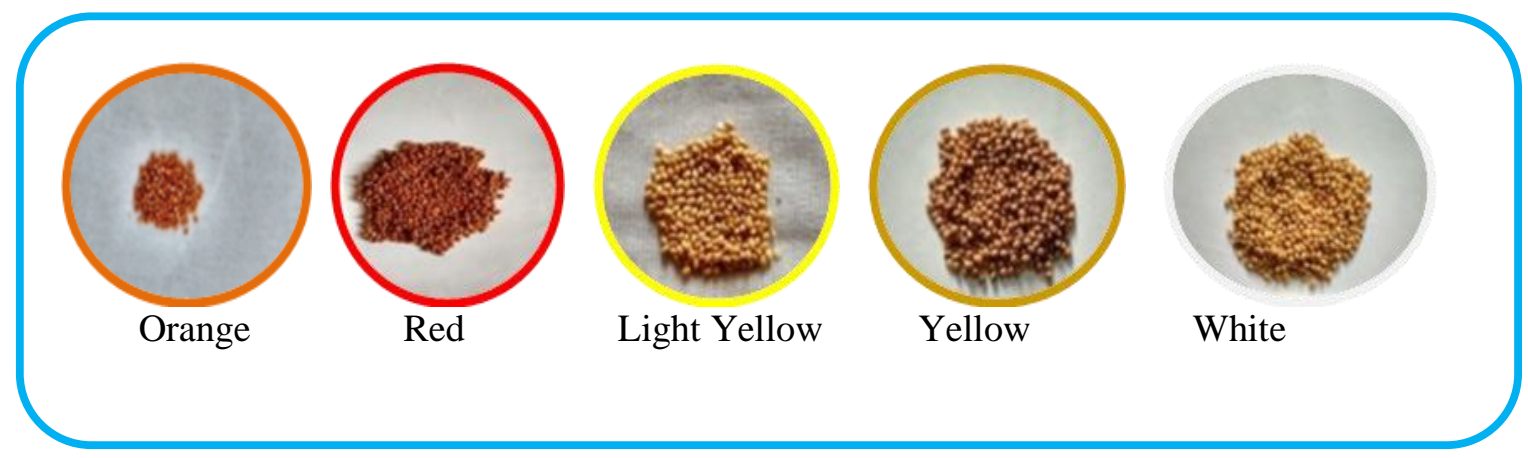


Fig.2 Quantitative characters

1. Flag leaf: Blade length $(\mathrm{cm})$

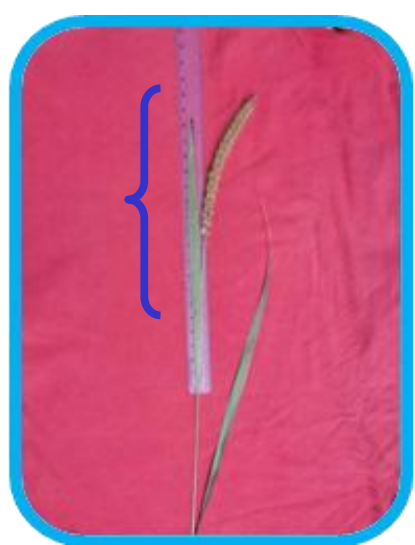

3. Peduncle length $(\mathrm{cm})$

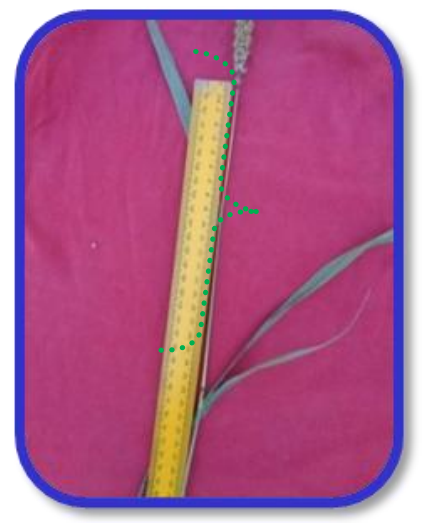

6. Plant height at Maturity $(\mathrm{cm})$

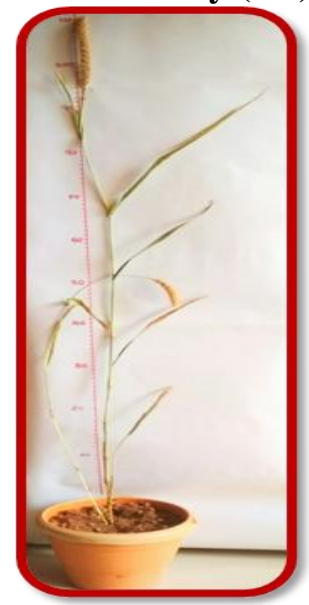

Medium (80-120 cm)

2. Flag leaf: Blade width (cm)

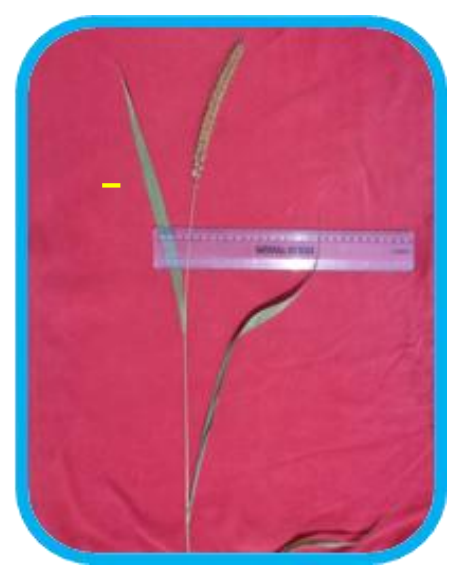

4. Peduncle exertion

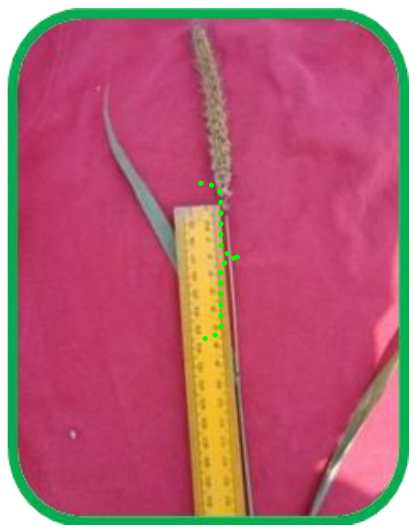

5. Panicle length $(\mathrm{cm})$

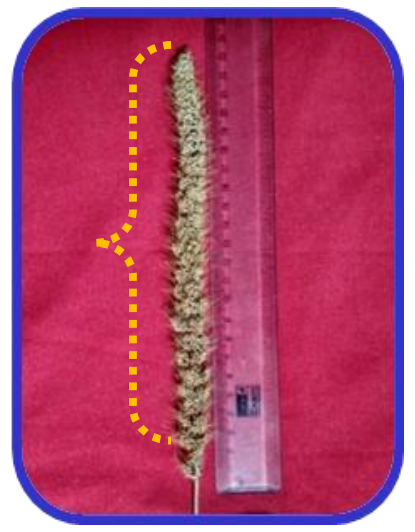

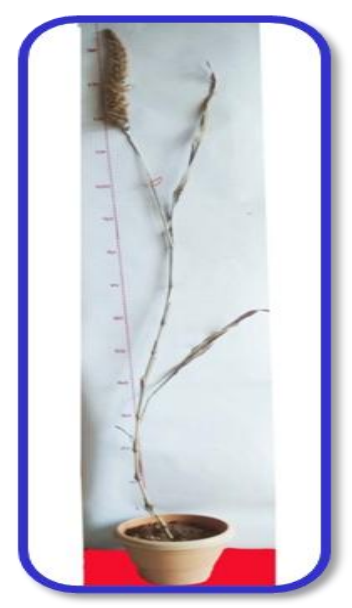

Tall $(>120 \mathrm{~cm})$ 
Fig.3 Morphological key for identification of 50 Italian millet germplasm accessions

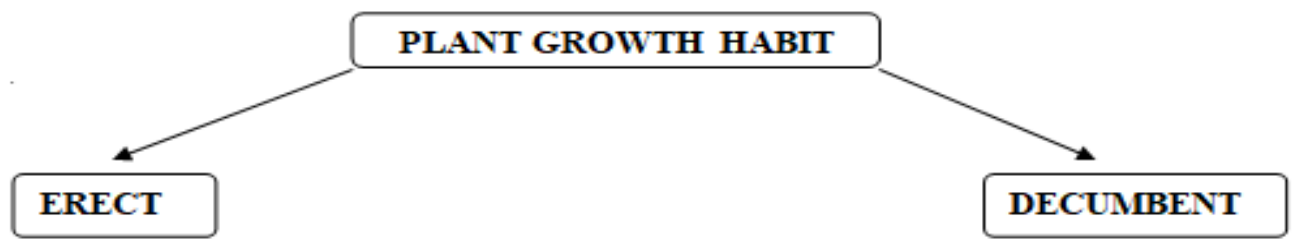

SiA 3328, SiA 3333,

SiA 3340, SiA 3346,

SiA 3384, SiA 3389,

SiA 3390 , SiA 3397 ,

SiA 3398.
SiA 3085, SiA 3156, SiA 3221, SiA 3322, SiA 3327, SiA 3347, SiA 3354,SiA 3355, SiA 3363, SiA 3364, SiA 3367, SiA 3369, SiA 3375, SiA 3376, SiA 3377, SiA 3378, SiA 3382, SiA 3383, SiA 3386, SiA 3392, SiA 3393, SiA 3395, SiA 3396, SiA 3399, SiA 3400, SiA 3401, SiA 3403, SiA 3404, SiA3318, SiA 3405, Srilakshmi, Suryanandi, Prasad, Narasimharaya, Krishnadeyaraya, ISC-379, SiA 3384, SiA 3407, SiA 3381, SiA 3447, SiA 3335, SiA 3394.
SiA 3221, SiA 3322, SiA 3327, SiA 3328, SiA 3333, SiA 3335, SiA 3340, SiA 3346, SiA 3347, SiA 3354, SiA 3355, SiA 3363, SiA 3364, SiA 3367, SiA 3369, SiA 3375, SiA 3376, SiA 3377, SiA 3381, SiA 3383, SiA 3384, SiA 3386, SiA 3389, SiA 3392, SiA 3390, SiA 3394, SiA 3395, SiA 3396,SiA 3397, SiA 3398, Prasad, Krishnadeyaraya SiA 3399, SiA 3400, SiA 3401, SiA 3403, SiA 3404, SiA 3318, SiA 3407, SiA 3405, SiA 3447, Suryanandi.

\section{PIGMENTED}

SiA 3085, SiA 3156, SiA 3378, SiA 3382, SiA 3393 Srilakshmi, Narasimharaxa and ISC 379. 


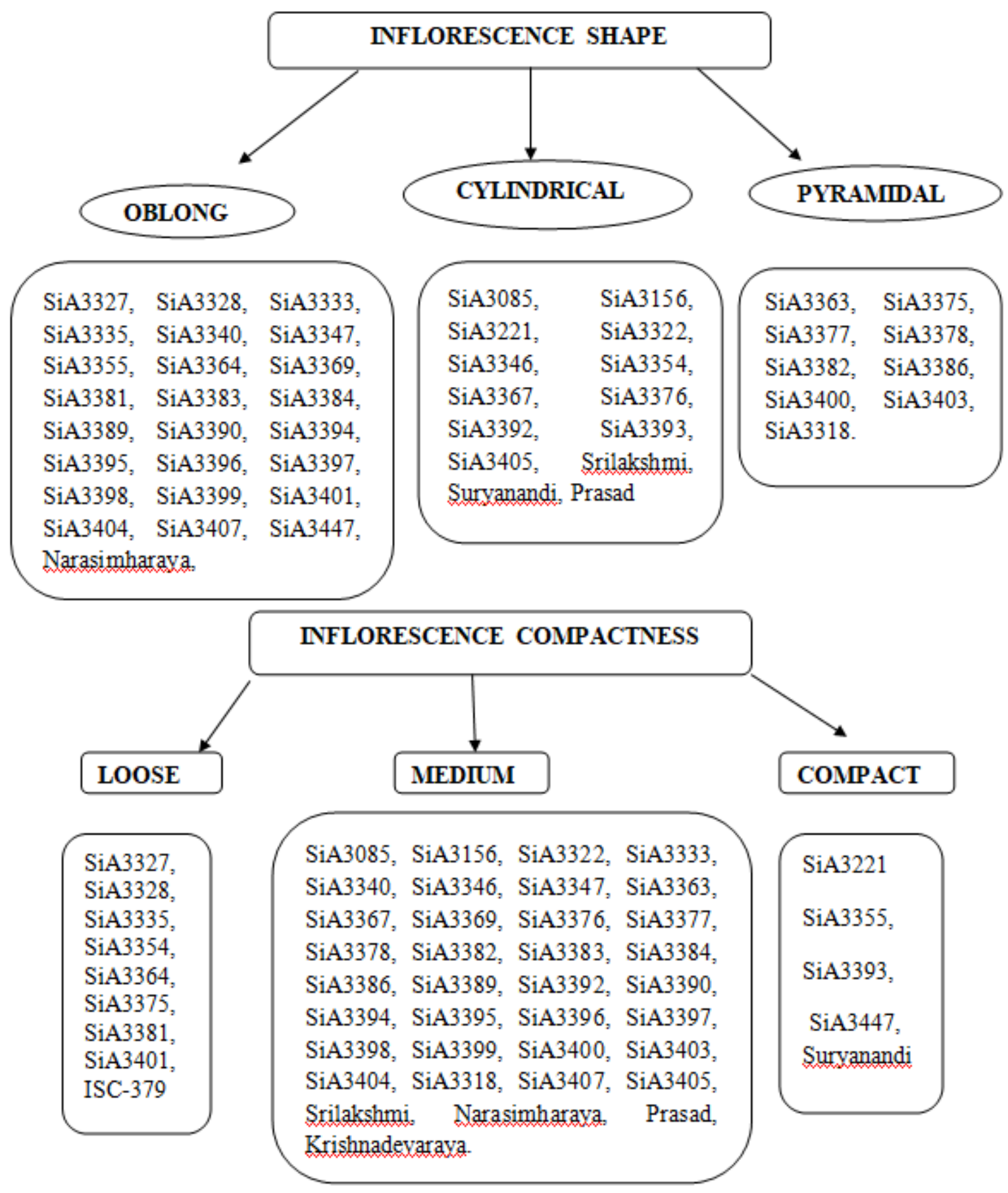




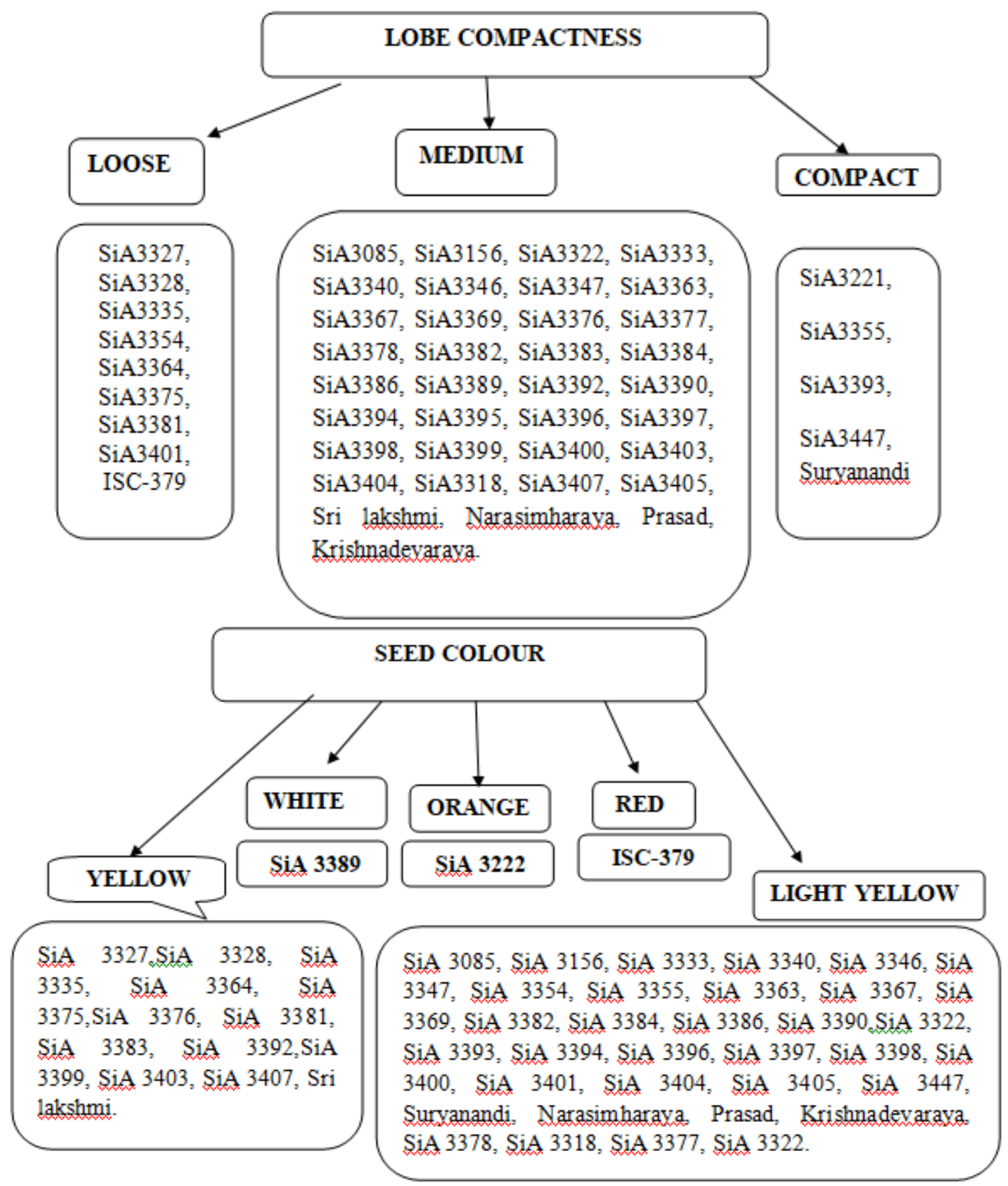

\section{Panicle length $(\mathrm{cm})$}

For the trait, panicle length, majority of the germplasm accessions reported long (46) while only few (4) were medium. Similar results were noted by Nirmala kumari et al., (2010) and Nehra et al., (2016) for this trait.

In conclusion, morphological characterization of 50 foxtail millet germplasm accessions for six qualitative traits revealed existence of ample polymorphism for the characters. It was observed that for plant growth habit, erect was dominant over decumbent; for plant pigmentation at auricle, non-pigmentation was dominant over pigmentation; for inflorescence shape, oblong was dominant over cylindrical and pyramidal; for inflorescence compactness, medium was dominant over compact and loose; for lobe compactness, presence was dominant over 
absence; for seed colour; yellow was dominant over light yellow, orange, red and white. Data analysis for six quantitative traits inferred medium for flag leaf blade length, flag leaf blade width, peduncle length; long for peduncle exertion and panicle length; tall for plant height. Based on the findings, it can be concluded that morphological DUS descriptors can be effectively used for identification, documentation and grouping of varieties along with their use in registration and unambiguous identification in the field. The morphological descriptors are of immense help to the plant breeders for selecting desirable genotypes easily through visual assessment and further the highly contrasting germplasm accessions for a particular trait can be utilized to develop mapping populations in order to map QTLs. Although DUS testing has been done precisely to provide diversity assessment at morphological level but there remains a further scope to validate the findings through diversity analysis at molecular level as molecular markers based genetic diversity analyses helps for more effective assessment of genetic relationship among the genotypes.

\section{Acknowledgements}

The authors are highly thankful to Acharya N.G. Ranga Agricultural University, Guntur, Andhra Pradesh, India for providing foxtail millet genetic resources and assistance for the conduct of experiment.

\section{References}

Ahmed, I., Molla, R. Md., Rohman, Motiar. Md. Ad. Hossain, A. Md. 2017. Morphological characterization of foxtail millet germplasm. International Journal of Applied Research. 3(2):1-9.

Amgai, R.B., Pantha, S., Chhetri, T.B., Budhathoki, S.K., Khatiwada, S.P and Mudwari. 2011. Variation on agromorphological traits in Nepalese foxtail millet (Setaria italica (L) P Beauv). Agronomy Journal of Nepal. 2:133-138.

Annual report, AICRP on Small Millets. 2016-17. Directorate of Economics and Statistics, Hyderabad-500004.

Banu, H., Gowda, J and Gowda, C.M.V. 2017.Genetic variability and multivariate analysis in reference set for yield characters in foxtail millet (Setaria italica (L.) Beauv). Green farming. 8(5): 1024-1028.

Murugan, R and Nirmala kumari, A. (2006). Genetic divergence in foxtail millet. (Setaria italica (L.) Beauv). Indian Journal of Genetics. 66(4):339- 340.

Nehra, M., Kumar, M., Vart, D., Sharma, R.K and Choudhary, M. 2016. DUS characterization and diversity assessment in pearl millet inbreds. Electronic Journal of Plant Breeding. 7(4): 925-933.

Nirmala kumari, A and Vetriventhan, M. 2010. Characterization of foxtail millet germplasm collections for yield contributing triats. Electronic Journal of Plant Breeding. 1(2): 140-147.

Reddy, G.V., Upadhyaya, H.D. and Gowda, C.L.L., 2006. Characterization of world's foxtail millet germplasm collections for morphological traits. International Sorghum and Millets Newsletter. 47: 107109.

Reddy, G.V., Upadhyaya, H.D., Gowda, C.L.L. and Singh, S. 2009. Characterization of eastern African finger millet germplasm for qualitative and quantitative characters at ICRISAT. SAT ejournal/ ejournal.icrisat.org. 3(1):1-4.

\section{How to cite this article:}

Amarnath, K., A.V.S. Durga Prasad and Chandra Mohan Reddy, C.V. 2019. DUS Characterization of Germplasm Accessions in Foxtail Millet (Setaria italica (L.) Beauv.). Int.J.Curr.Microbiol.App.Sci. 8(02): 2410-2422. doi: https://doi.org/10.20546/ijcmas.2019.802.281 